\title{
ON THE BREAK-UP OF INVARIANT TORI WITH THREE FREQUENCIES
}

\author{
J.D. MEISS \\ Program in Applied Mathematics \\ University of Colorado \\ Boulder, $\mathrm{CO}$
}

\begin{abstract}
We construct an approximate renormalization operator for a two and one half degree of freedom Hamiltonian corresponding to an invariant torus with a frequency in the cubic field $\mathrm{Q}(\tau)$, where $\tau^{3}+\tau^{2}-2 \tau-1=0$. This field has irrational vectors that are most robust in the sense of supremal Diophantine constant. Our renormalization operator has a critical fixed point, but it is not hyperbolic. Instead it has a codimension three stable manifold with one unstable eigenvalue, $\delta \approx 2.88$, and two neutral eigenvalues.
\end{abstract}

\section{Introduction}

A major open problem in the study of Hamiltonian dynamics is the mechanism for the break-up of invariant tori in systems with more than two frequencies. The two frequency case, though not completely solved has seen many advances in the past 15 years, including Aubry-Mather theory [1;2], renormalization theory [3], converse KAM theory $[4 ; 5]$, and the anti-integrable limit $[6 ; 7]$.

The story has the following plot outline: beginning with an integrable system of 2 degrees of freedom, KAM theory implies that almost all invariant tori (those with Diophantine frequency ratios) are stable to perturbation. However, every invariant two torus is eventually destroyed by strong enough perturbation (converse KAM) and is replaced by a "cantorus," a torus with a cantor set cross-section. The tori that are locally most robust are the noble irrationals - those whose frequency ratios are equivalent to the golden mean under a modular transformation. At a critical point a noble torus exhibits a self-similar structure, and this local picture is universal. The destruction of tori of more general frequencies can also be described in a local way with a renormalization operator, but the picture is not strictly self-similar, though universality is expected to hold except possibly for frequencies not of "constant type."

Some of these results can be generalized to more dimensions. KAM theory implies that Diophantine tori are stable. Generalizations of converse KAM theory [8] and the anti-integrable limit [7] can be developed. However, very little is understood about the way in which the torus-to-cantorus transition occurs. In this note, we review our attempt to understand this transition by the construction of an approximate renormalization theory [9].

We focus on the case of a periodically time dependent Hamiltonian $H(x, y, u, v, t)$. where $(x, y, u, v) \in T^{2} \times R^{2}$. Here $(x, y)$ is the configuration and $(u, v)$ the momentum. We claim there are three essential parameters needed to study such a system, and these can be 
taken to be amplitudes of potential energy terms. Our model is a point particle in the plane acted on by the field of three traveling waves, which has the Hamiltonian

$$
H=\frac{1}{2}(u, v) \cdot\left(\begin{array}{ll}
\alpha & \beta \\
\beta & \gamma
\end{array}\right) \cdot\left(\begin{array}{l}
u \\
v
\end{array}\right)+A \cos (2 \pi x)+B \cos (2 \pi k y)+C \cos (2 \pi d(t-x-y))
$$

Without loss of generality, the wavenumbers $(\mathrm{k}, \mathrm{l})$ can be taken to be positive and the energy can be scaled so that the mass matrix has unit determinant, $\alpha \gamma-\beta^{2}=1$

\section{Tori and Frequencies}

We study the rotational tori, that is tori homotopic to the constant momentum tori. The frequency vector $\omega$ is the average direction that an orbit moves around the torus (assuming this limit exists). We let $\omega \in \mathrm{R}^{3}$, where the first component gives the periodic time dependence, and the length of the vector is unimportant. A frequency is commensurate if there is a nonzero integer vector $m$ such that $m \cdot \omega=0$. Such a relation is a resonance condition. If $\omega$ has no resonances then it is incommensurate. If $\omega$ has $d$ independent resonances, then it is proportional to an integer vector. The Diophantine constant for $\omega$ is defined by $C^{\tau}(\omega)=\liminf _{m \rightarrow \infty}\|m\|^{\tau}|m \cdot \omega|$ where $\|m\|=\max \left(\left|m_{i}\right|\right)$. A Diophantine frequency has $\mathrm{C}^{\mathrm{d}}(\omega) \neq 0$.

The theory of simultaneous approximation of frequency vectors is not as complete as continued fraction theory; see, e.g., [10]. We adopt a Farey approximation technique proposed in [11]. Begin with three resonances $\mathrm{m}_{1}=(1,0,0), \mathrm{m}_{2}=(0,1,0), \mathrm{m}_{3}=(0,0,1)$, each corresponding to a plane in $\mathrm{R}^{3}$. The set of three resonances delineates a cone (the positive octant) denoted by the matrix $M=\left(\mathrm{m}_{1}, \mathrm{~m}_{2}, \mathrm{~m}_{3}\right)^{\mathrm{t}}$.

To construct the Farey sequence for an win $\mathrm{M}$, divide the cone using the new resonance $\mathrm{m}^{\prime}=\mathrm{m}_{1}-\mathrm{m}_{2}$. There is now a right cone $\mathrm{M}_{\mathrm{R}}=\left(\mathrm{m}_{3}, \mathrm{~m}^{\prime}, \mathrm{m}_{2}\right)^{\mathrm{t}}$, and a left cone $M_{L}=\left(-m^{\prime}, m_{3}, m_{1}\right)^{t}$. If $\omega \in M_{L}$, record an $L$, otherwise permute the first two coordinates and record a $\mathrm{P}$. Repetition of this transformation gives a sequence of $\mathrm{L}$ and $\mathrm{P}$ 's. The operations can be represented by linear transformations, $\mathrm{M}_{\mathrm{S}}=\mathrm{S}^{-1} \mathrm{M}$, where

$$
S=\left\{\begin{array}{ll}
P & \text { if }\left(m_{1}-m_{2}\right) \cdot \omega>0 \\
L & \text { if }\left(m_{1}-m_{2}\right) \cdot \omega \leq 0
\end{array}, \quad P=\left(\begin{array}{lll}
0 & 1 & 0 \\
1 & 0 & 0 \\
0 & 0 & 1
\end{array}\right), \quad L=\left(\begin{array}{lll}
0 & 0 & 1 \\
1 & 0 & 1 \\
0 & 1 & 0
\end{array}\right)\right.
$$

Note that $\operatorname{det}(\mathrm{P})=\operatorname{det}(\mathrm{L})=1$ so $\operatorname{det}\left(\mathrm{M}_{\mathrm{S}}\right)=1$. We can think of $\omega$ as the sequence $\mathrm{S}_{1} \mathrm{~S}_{2} \mathrm{~S}_{3} \ldots$ If $\omega$ is an integer vector (with no common factors) then this sequence eventually terminates [12].

The "simplest" incommensurate frequency vectors have periodic Farey sequences. When the period is $q, \omega$ is the eigenvector with largest eigenvalue of the nonnegative matrix $\mathrm{S}_{1} \ldots \mathrm{S}_{\mathrm{q}}$. This implies that the components of $\omega$ are elements of a (possibly reducible) cubic field. The simplest of these is the eigenvector of $L,\left(1, \sigma^{2}, \sigma\right)$, where $\sigma \approx$ 1.324717957 is the real solution of $\sigma^{3}=\sigma+1$, called the spiral mean. This vector is an integral basis for the cubic field, $\mathrm{Q}(\sigma)$, generated by $\sigma$. 
If we look at products of the matrices $\mathrm{L}$ and $\mathrm{P}$, the next periodic sequence that gives an irreducible cubic irrational is of period four, containing three $\mathrm{L}$ factors and one P. Each of these sequences generates a frequency in the cubic field, $Q(\tau)$, where $\tau=$ $2 \cos (2 \pi / 7) \approx 1.2469796$ is a solution of $\tau^{3}+\tau^{2}-2 \tau-1=0$. One such matrix is

$$
\mathrm{LPL}^{2}=\left(\begin{array}{lll}
1 & 0 & 1 \\
1 & 1 & 2 \\
0 & 1 & 0
\end{array}\right), \quad \lambda=1+\tau, \quad \omega=\left(1, \tau^{2}+\tau, \tau\right)
$$

where $\lambda \approx 2.246979604$ is the only eigenvalue of LPL ${ }^{2}$ bigger than one. The cubic field $\mathrm{Q}(\tau)$ is the totally real cubic field with minimal discriminant $(\Delta=43)$ and $\omega$ is an integral basis for $\mathrm{Q}(\tau)$. Numbers in this cubic field appear to be the generalization of the golden mean -in the sense of having the largest possible Diophantine constant. It is known that $\sup _{\omega} C^{2}(\omega) \geq 2 / 7$ and numerical evidence indicates that $2 / 7$ is indeed the supremum [13]. Using a reasonable conjecture about the continued fraction of $\tau$, Cusick has shown that there are integral bases in $\mathrm{Q}(\tau)$ whose Diophantine constants limit on $2 / 7$ [14]. If $\tau$ is the correct generalization of the golden mean, we would expect some equivalence class of frequencies in $\mathrm{Q}(\tau)$ to give the most robust tori [15]. The set of frequencies with a given tail can be thought of as equivalent under the operation of stripping off the head of the sequence. All such frequencies will have the same renormalization behavior.

Preliminary investigations of $\mathrm{Q}(\tau)$ show that it contains frequencies with two distinct periodic Farey tails, $\mathrm{LPL}^{2}$ as above and $(\mathrm{LP})^{6} \mathrm{~L}$. In addition there appear to be elements of the cubic field with aperiodic Farey sequences. We do not understand the significance of these. As a final remark, Arnold's simplicial generalization of the continued fraction $[16 ; 17]$ also points to the field $\mathrm{Q}(\tau)$ as the "simplest" (Arnold, private communication).

\section{Renormalization Transformation}

The renormalization focuses on a region of phase space in which orbits of a given frequency ratio are expected. We define two such transformations corresponding to the $\mathrm{L}$ and $\mathrm{P}$ Farey steps. These are obtained by formally assuming that each of the parameters $\mathrm{A}, \mathrm{B}, \mathrm{C}=O(\varepsilon)$, obtaining a canonical transformation to eliminate one of the resonances to $O\left(\varepsilon^{2}\right)$, and then transform the new Hamiltonian back to its original form [9].

For the $\mathrm{L}$ transformation, we eliminate the $\mathrm{m}_{2}=(010)$ resonance by a near identity canonical transformation. The final transformation consists of the maps

$$
\begin{gathered}
\mathrm{L}:\left\{\mathrm{k}^{\prime}=\frac{\ell}{\mathrm{k}}, \boldsymbol{\ell}=\frac{1}{1+\mathrm{k}}\right. \\
\mathrm{L}:\left(\begin{array}{c}
\alpha^{\prime} \\
\beta^{\prime} \\
\gamma^{\prime}
\end{array}\right)=\frac{1}{1+\mathrm{k}}\left(\begin{array}{ccc}
1 / \mathrm{k} & -2 & \mathrm{k} \\
1 & 1-\mathrm{k} & -\mathrm{k} \\
\mathrm{k} & 2 \mathrm{k} & \mathrm{k}
\end{array}\right)\left(\begin{array}{l}
\alpha \\
\beta \\
\gamma
\end{array}\right)
\end{gathered}
$$




$$
L:\left\{A^{\prime}=\frac{(1+k)^{3} \beta}{2 k^{2}} A B, B^{\prime}=\frac{1+k}{k} C, C^{\prime}=\frac{1+k}{k} A\right.
$$

This is the approximate renormalization operator. The permutation $\mathrm{P}$ corresponds to the involution

$$
\mathrm{P}:(\mathrm{k}, \mathbf{l}, \alpha, \beta, \gamma, \mathrm{A}, \mathrm{B}, \mathrm{C}) \rightarrow\left(\frac{1}{\mathrm{k}}, \frac{\mathbf{l}}{\mathrm{k}}, \gamma, \beta, \alpha, \mathrm{B}, \mathrm{A}, \mathrm{C}\right)
$$

\section{Renormalization for $Q(\tau)$}

For the frequency (4) we construct the map $\mathcal{L P L} \mathcal{L}^{2}$. For the wavenumbers this gives

$$
\mathrm{LPL}^{2}:(k, \boldsymbol{l}) \rightarrow\left(\frac{\mathrm{k}}{\mathrm{k}+\boldsymbol{l}}, \frac{\mathrm{k}}{\mathrm{I}+\mathrm{k}+\mathrm{k}}\right)
$$

It has a unique fixed point, $(\mathrm{k}, \mathrm{l})=\left(\tau^{2}-1,2-\tau^{2}\right)$, in the positive quadrant. This fixed point is a stable node with eigenvalues $\lambda=0.247$ and -0.357 , and is a global attractor for the positive quadrant.

Since the wavenumber map is contracting, the wavenumber dynamics is nonessential, and we therefore evaluate the mass map at the fixed point $\mathrm{k}=\tau^{2}-1$ giving the linear map

$$
\mathrm{LPL}^{2}: \frac{1}{\tau(\tau+1)}\left(\begin{array}{ccr}
1 & 2 & 1 \\
1+\tau^{-1} & -\tau^{-1} & -1 \\
2+\tau & -2\left(1+\tau^{-1}\right) & 1
\end{array}\right)
$$

Recall that this operator preserves the subspace $\alpha \gamma-\beta^{2}=1$. In fact this matrix is a square root of the identity; it has eigenvalues $(-1,1,1)$ and is diagonalizable. Therefore this map is not contracting - in general the mass matrix oscillates with period two, unless the component along the first eigenvector is zero, i.e., when the mass is chosen such that (1$\left.\tau-\tau^{2}\right) \alpha+2 \beta+\gamma=0$. Otherwise the general orbit of $\beta$ is of the form

$$
\beta_{\mathrm{n}}=\mathrm{c}_{0}+(-1)^{\mathrm{n}} \mathrm{c}_{1}
$$

This violates the notion of "universality": asymptotics of the orbit under the renormalization depend on the parameters of the initial Hamiltonian.

The parameter map depends on the wavenumber $\mathrm{k}$ and the mass matrix through $\beta$. Consider first the case when $\mathrm{c}_{1}=0$, so that $\beta$ is fixed:

$$
L P L^{2}:(A, B, C) \rightarrow\left(\frac{\tau^{24}}{8\left(\tau^{2}-1\right)^{9}} \beta^{3} A^{2} B C, \frac{\tau^{10}}{2\left(\tau^{2}-1\right)^{4}} \beta A B, \frac{\tau^{6}}{\left(\tau^{2}-1\right)^{3}} A\right)
$$

There are two fixed points, $\mathrm{A}=\mathrm{B}=\mathrm{C}=0$ - the KAM fixed point, and the critical fixed point: 


$$
A_{c}=B_{c}=\frac{2\left(\tau^{2}-1\right)^{4}}{\tau^{10} \beta}, \quad C_{c}=\frac{2\left(\tau^{2}-1\right)}{\tau^{4} \beta}
$$

The KAM fixed point is attracting. The critical point can be studied by taking the log of the amplitude map to give, in terms of $\mathrm{a}=\log (\mathrm{A}), \mathrm{b}=\log (\mathrm{B}), \mathrm{c}=\log (\mathrm{C})$, the affine map

$$
\left(\begin{array}{l}
a^{\prime} \\
b^{\prime} \\
c^{\prime}
\end{array}\right)=\left(\begin{array}{lll}
2 & 1 & 1 \\
1 & 1 & 0 \\
1 & 0 & 0
\end{array}\right)\left(\begin{array}{l}
a \\
b \\
c
\end{array}\right)+v_{0}
$$

Thus stability is governed by the linear matrix above. This matrix has the characteristic polynomial $\lambda^{3}-3 \lambda^{2}+1=0$ (not related to $\tau$ ), so that

$$
\lambda_{1}=\delta \approx 2.8793852, \lambda_{2} \approx .65270365, \lambda_{3} \approx-.53208888
$$

Thus there is a one dimensional unstable manifold, and a two dimensional stable set.

For the general case, $\beta$ is not fixed, and the amplitude map is periodically forced. However, for the amplitude dynamics, there is still a two dimensional center-stable manifold that has a one dimensional unstable manifold.

\section{Conclusion}

The approximate renormalization for the cubic $\tau$ is qualitatively quite similar to that we found for the spiral mean, $\sigma$. The only real difference is that the mass matrix for $\sigma$ undergoes an irrational rotation, while for $\tau$ we found period two orbits. However, both of these are structurally unstable, and we would expect the dynamics of the exact renormalization to be more complicated. What should be preserved is the codimension one critical surface containing a fixed point and a $2 \mathrm{D}$ normally hyperbolic invariant manifold, with 1 unstable normal direction with eigenvalue $\delta \approx 2.88$, the remaining normal directions being attracting.

As for the spiral mean case, we find that a typical one parameter system is not "self-similar" at criticality. Instead properties of the system such as the stability parameters of periodic orbits (i.e., the residues) are predicted to oscillate

It will be interesting to investigate the question of "robustness" of tori with various frequencies by examining the relation between the critical surfaces for various frequencies.

\section{Acknowledgments}

This paper is the report of collaborative work with Robert MacKay and Jaroslav Stark. Our collaboration was supported by a UK Science and Engineering Research Council Visiting Fellowship grant and a NATO Scientific Affairs Division Collaborative Research grant (\#921181). I would also like to acknowledge the support of the National Science Foundation under grant \#DMS-9305847. 


\section{References}

[1] Mather, J. N. (1982) Existence of Quasi-periodic Orbits for Twist Homeomorphisms of the Annulus, Topology 21 pp. 457-467.

[2] Aubry, S. and P. Y. Le Daeron (1983) The Discrete Frenkel-Kontorova Model and Its Extensions, Physica 8D pp. 381-422.

[3] MacKay, R. S. (1993)Renormalisation in Area-Preserving Maps, World Scientific, Singapore.

[4] MacKay, R. S. and I. C. Percival (1985) Converse KAM: Theory and Practice, Comm. Math. Phys. 98 pp. 469-512.

[5] MacKay, R. S. (1989) A Criterion for Non-existence of Invariant Tori for Hamiltonian Systems, Physica 36D pp. 64-82.

[6] Aubry, S. and G. Abramovici (1990) Chaotic Trajectories in the Standard Map, the Concept of Anti-integrability, Physica D 43 pp. 199-219.

[7] MacKay, R. S. and J. D. Meiss (1992) Cantori for Symplectic Maps near the Antiintegrable Limit, Nonlinearity 5 pp. 149-160.

[8] MacKay, R. S., J. D. Meiss, et al. (1989) Converse KAM Theory for Symplectic Twist Maps, Nonlinearity 2 pp. 555-570.

[9] MacKay, R. S., J. D. Meiss, et al. (1994) An Approximate Renormalization for the Break-up of Invariant Tori with Three Frequencies, Physics Letters A 190 pp. 417424.

[10] Cassels, J. W. S. (1965)An Introduction to Diophantine Approximation, Cambridge University Press, Cambridge.

[11] Kim, S. and S. Ostlund (1986) Simultaneous Rational Approximations in the Study of Dynamical Systems, Phys. Rev. A 34 pp. 3426-3434.

[12] Baesens, C., J. Guckenheimer, et al. (1991) Three Coupled Oscillators: Modelocking, Global Bifurcations and Toroidal Chaos, Physica 49D pp. 387-475.

[13] Szerkeres, G. (1985) Simultaneous Approximation Constant, Ars Combinatoria 19A pp. 237-243.

[14] Cusick, T. W. (1974) The Two-Dimensional Diophantine Approximation Constant, Monatshefte für Mathematik 78 pp. 297-304.

[15] Lochak, P. (1992) Canonical Perturbation Theory via Simultaneous Approximation, Russian Mathematical Surveys 47 pp. 59.

[16] Lachaud, G. (1993) Polyèdre d'Arnol'd et voile d'un cône simplicial: analogues du théorème de Lagrange, C.R.Acad. Sci. Paris, Série I 317 pp. 711-716.

[17] Elena, K. (1994) La Pérodicité des Fractions Continues Multidimensionnelles, C.R.Acad. Sci. Paris, Série I 319. 